\title{
$4 \frac{40}{42} \mid 94<2$ \\ PREPARED FOR THE U.S. DEPARTMENT OF ENERGY, UNDER CONTRACT DE-AC02-76-CHO-3073
}

PPPL-2982

UC-427

PPPL-2982

THE VALIDITY OF THE EXTENDED ENERGY PRINCIPLE

BY

M.S. CHANCE, J.L. JOHNSON AND R.M. KULSRUD

APRIL, 1994
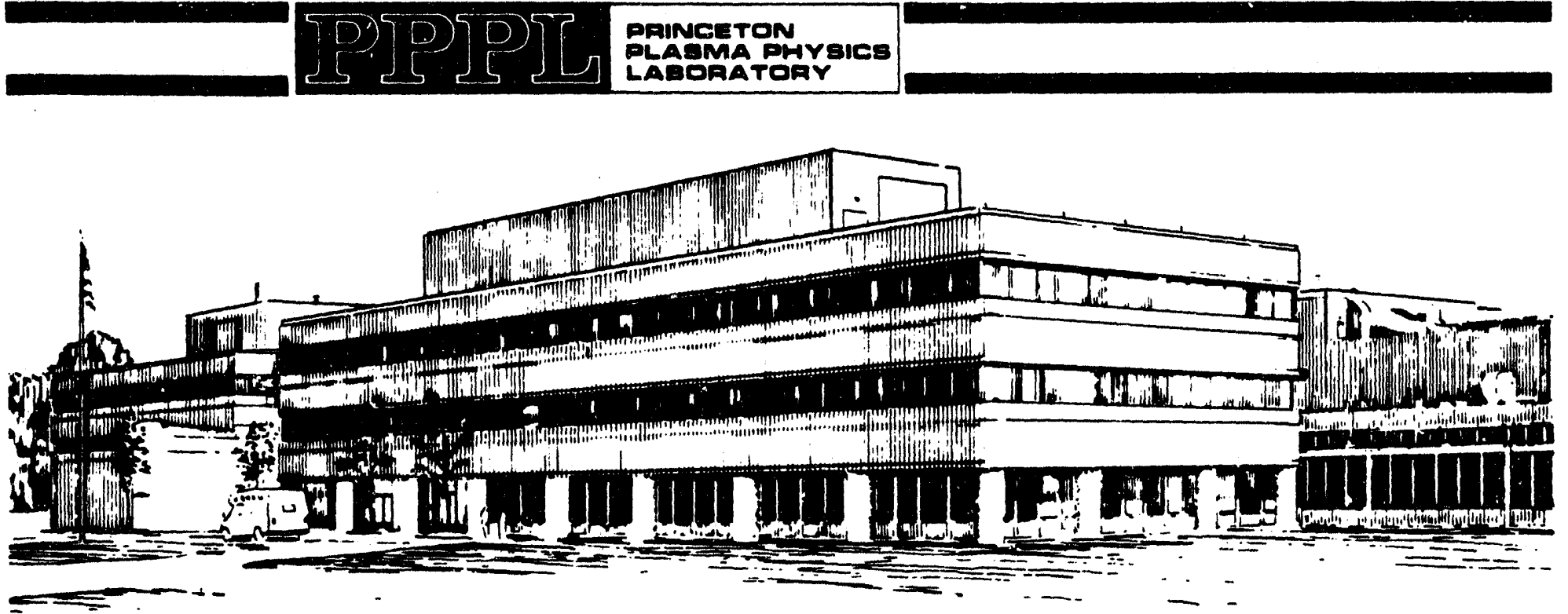

PRINCETON UNIVERSITY, PRINCETON, NEW JERSEY

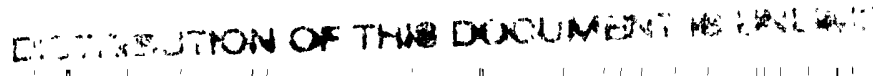


NOTICE

This report was prepared as an account of work sponsored by an agency of the United States Government. Neither the United States Government nor any agency thereof, nor any of their employees, makes any warranty, express or implied, or assumes any legal liability or responsibility for the accuracy, completeness, or usefulness of any information, apparatus, product, or process disclosed, or represents that its use would not infringe privately owned rights. Reference herein to any specific commercial produce, process, or service by trade name, trademark, manufacturer, or otherwise, does not necessarily constitute or imply its endorsement, recommendation, or favoring by the United States Government or any agency thereof. The views and opinions of authors expressed herein do not necessarily state or reflect those of the United States Government or any agency thereof.

\section{NOTICE}

This report has been reproduced from the best available copy. Available in paper copy and microfiche.

Number of pages in this report: 14

DOE and DOE contractors can obtain copies uf this report from:

Office of Scientific and Technical Information

P.O. Box 62

Oak Ridge, TN 37831 ;

(615) 576-8401.

This report is publicly available from the:

National Technical Information Service

Department of Commerce

5285 Port Royal Road

Springfield, Virginia 22161

(703) $487-4650$ 


\title{
The Validity of the Extended Energy Principle
}

\author{
M. S. Chance, J. L. Johnson, and R. M. Kulsrud \\ Plasma Physics Laboratory, Princeton University \\ P. O. Box 451, Princeton New Jersey 08543
}

\begin{abstract}
A recent analysis of plasma stability based on modifications of the extended energy principle for magnetohydrodynamic stability led to conclusions that are too optimistic. The original interpretation of this principle is indeed applicable. The present analysis demonstrates explicitly the fallacy of using the wrong functional for $\delta W$ in the extended energy principle. It then shows that the original energy principle functional $\delta W_{\mathrm{B}}$ is also obtained for a model in which a surface mass is incorporated to provide pressure balance. This work therefore indicates, but does not prove, that the eigenfunctions that are obtained from a minimization of the extended energy principle with the proper kinetic energy norm provide a good representation of what would be achieved with an exact treatment.
\end{abstract}




\section{Introduction}

Lehnert [1991,1992, 1993] recently called into question the validity of the energy principle for MHD stability in its extended form [Bernstein et al., 1958]. In this note we address his criticism and show that it is flawed. In addition we point out some physical ideas related to the extended energy principle and make some comments on its application in computational MHD stability studies which use the PEST code [Grimm et al., 1976].

The difficulty raised by Lehnert is really quite simple from a mathematical point of view. We first sketch out his argument and the fallacy in it.

In the Bernstein et al. paper, it was shown that the potential energy which is a functional $\delta W_{\mathrm{B}}(\boldsymbol{\xi}, \boldsymbol{\xi})$ of arbitrary virtual displacements $\boldsymbol{\xi}$ gives a simple necessary and sufficient condition for stability. The authors distinguished between "proper" perturbations $\tilde{\boldsymbol{\xi}}$ and "improper" perturbations $\boldsymbol{\xi}$, according to whether the $\boldsymbol{\xi}$ when coupled with the perturbed vacuum field $\boldsymbol{A}$ led to pressure balance on the plasma-vacuum interface or not. For proper perturbations, $\delta W_{\mathrm{B}}(\tilde{\boldsymbol{\xi}}, \tilde{\boldsymbol{\xi}})$ is the perturbed potential energy $-\frac{1}{2} \int_{\mathrm{P}} d \tau \tilde{\boldsymbol{\xi}} \cdot \boldsymbol{F}(\tilde{\boldsymbol{\xi}})$ where $\boldsymbol{F}$ is the perturbed force. For improper $\boldsymbol{\xi}$ 's, $\delta W_{\mathbf{B}}(\boldsymbol{\xi}, \boldsymbol{\xi})$ does not have any immediate significance, but Bernstein et al. were able to show that if $\delta W_{\mathrm{B}}(\boldsymbol{\xi}, \boldsymbol{\xi})$ were negative for an improper $\boldsymbol{\xi}$ the equilibrium would be unstable.

It is the last statement whose validity is challenged by Lehnert. Now, it is important to realize that there are many different functionals $\delta W(\boldsymbol{\xi}, \boldsymbol{\xi})$ that are equal over the restricted class of proper perturbations $\tilde{\boldsymbol{\xi}}$, but that differ over the wider class of improper $\boldsymbol{\xi}$ 's. In particular, although he acknowledges the existence of the functional $\delta W_{\mathrm{B}}(\boldsymbol{\xi}, \boldsymbol{\xi})$, Lehnert's argument is based on the functional $\delta W_{\mathrm{L}}(\boldsymbol{\xi}, \boldsymbol{\xi})=-\frac{1}{2} \int_{\mathrm{P}} d \tau \boldsymbol{\xi} \cdot \boldsymbol{F}(\boldsymbol{\xi})$ which is not equal to $\delta W_{\mathrm{B}}(\boldsymbol{\xi}, \boldsymbol{\xi})$ for an improper $\boldsymbol{\xi}$. In deriving their functional $\delta W_{\mathrm{B}}(\tilde{\boldsymbol{\xi}}, \tilde{\boldsymbol{\xi}})$ for a proper $\overline{\boldsymbol{\xi}}$, Bernstein et al. integrated by parts and made use of the pressure balance condition (which of course is not satisfied for an improper $\boldsymbol{\xi}$ ). They then simply extended the definition of this functional to all $\boldsymbol{\xi}$ 's by keeping the same form.

Lehnert in his papers shows that the extended energy principle does not apply to the form $\delta W_{\mathrm{L}}(\boldsymbol{\xi}, \boldsymbol{\xi})$. That is, if $\delta W_{\mathrm{L}}(\boldsymbol{\xi}, \boldsymbol{\xi})$ is negative for an improper $\boldsymbol{\xi}$ one need not have instability. This is of course true. But since he is discussing a functional which is essentially different from the functional $\delta W_{\mathrm{B}}(\boldsymbol{\xi}, \boldsymbol{\xi})$ employed in Bernstein et al.'s extended energy principle, his crit- 
icism is not valid.

One can of course ask for the significance of the functional $\delta W_{\mathbf{B}}(\boldsymbol{\xi}, \boldsymbol{\xi})$ for an improper $\boldsymbol{\xi}$. The functional $\delta W_{\mathrm{L}}(\boldsymbol{\xi}, \boldsymbol{\xi})$ would, if it is integrated over the plasma volume as in the derivation of $\delta W_{\mathrm{B}}$, contain a surface term $\delta W_{U}(\boldsymbol{\xi}, \boldsymbol{\xi})$ which is non zero for an improper $\boldsymbol{\xi}$. This term would cancel the contribution from a surface current, $\delta W_{\mathrm{K}}(\boldsymbol{\xi}, \boldsymbol{\xi})$, which Lehnert introduced to satisfy pressure balance, so that when he corrected his extended energy principle he should have recovered $\delta W_{\mathrm{B}}$. In this sense $\delta W_{\mathrm{B}}(\boldsymbol{\xi}, \boldsymbol{\xi})$ is the "true" work done to set up the improper perturbation. It is useful to note that Bernstein et al. said very clearly in their paper that $\delta W_{\mathrm{L}}$ may differ for improper perturbations from $\delta W_{\mathrm{B}}$ by the addition of terms which represent the work done at the surface against the unbalanced total pressure, so that $\delta W_{\mathrm{B}}$ must be used for the extended energy principle.

\section{Formulation}

The study of the stability of a magnetohydrodynamic equilibrium with respect to small perturbations has been important for the design and interpretation of experiments in the magnetic fusion energy program from its infancy. The proof of the self adjointness of the potential energy $\delta W=$ $-\frac{1}{2} \int_{\mathrm{P}} d \tau \tilde{\boldsymbol{\xi}} \cdot \boldsymbol{F}(\tilde{\boldsymbol{\xi}})$ with $\boldsymbol{F}(\tilde{\boldsymbol{\xi}})$ the perturbed force showed that ideal modes are purely growing or oscillatory in time and reduced the determination of stability to a simple variational problem. Recognition that the test functions for such a determination need not satisfy the boundary condition of pressure continuity at the plasma-vacuum interface further simplified the problem. In this section, we investigate the expression for the extended energy principle, which was derived by the Princeton group [Bernstein et al., 1958] and restated by Lehnert [1991, 1992, 1993]. Lehnert tries to argue that the forces due to a surface current associated with pressure balance can make the configuration stable even if an improper $\boldsymbol{\xi}$ that does not satisfy pressure balance at the plasma-vacuum interface makes $\delta W_{\mathrm{B}}$ of the extended energy principle negative. This is wrong. Bernstein et al. [1958] showed this by displaying a nearby proper $\tilde{\xi}$ which also makes $\delta W_{\mathrm{B}}$ negative while satisfying the pressure balance condition. Here we describe the modification of the perturbation which was made in the original energy principle paper to emphasize that the current which must be introduced to provide pressure balance does 
not change the stability criterion. We then examine Lehnert's model, which utilizes a different functional $\delta W_{\mathrm{L}}$ from that of the Energy Principle paper $\delta W_{\mathrm{B}}$, and show it really gives similar results if properly evaluated. To be specific, the effect of the surface current that must be introduced into $\delta W_{\mathrm{L}}$ for improper $\boldsymbol{\xi}$ 's that do not satisfy pressure balance is exactly what is necessary to cancel the surface term that arises from an integration by parts, but is neglected by Lehnert. We finish this section by considering an extended energy principle in which there is a finite surface mass at the interface, such that acceleration of the surface can sustain a pressure imbalance. We can argue from a comparison of the results of this model with those where a surface current is present that the eigenfunctions associated with a minimization of the $\delta W$ of the energy principle paper [Bernstein et al., 1958] with the proper kinetic energy normalization are, indeed, close to what would be obtained from a direct integration of the force equation.

We consider perturbations $\tilde{\boldsymbol{\xi}}$ about a plasma equilibrium satisfying the force balance equation $\boldsymbol{J} \times \boldsymbol{B}=\nabla p$. Thus

$$
\begin{gathered}
\rho \frac{\partial^{2} \tilde{\boldsymbol{\xi}}}{\partial t^{2}}=\boldsymbol{F}(\tilde{\boldsymbol{\xi}}), \\
\boldsymbol{F}(\tilde{\boldsymbol{\xi}})=(\nabla \times \tilde{\boldsymbol{Q}}) \times \boldsymbol{B}+\boldsymbol{J} \times \tilde{\boldsymbol{Q}}+\tilde{\boldsymbol{\xi}} \cdot \nabla p+\gamma p \nabla \cdot \tilde{\boldsymbol{\xi}}, \\
\tilde{\boldsymbol{Q}}=\boldsymbol{\nabla} \times(\tilde{\boldsymbol{\xi}} \times \boldsymbol{B}),
\end{gathered}
$$

with the conditions at the plasma-vacuum boundary

$$
\begin{gathered}
n \times \tilde{\boldsymbol{A}}=-(\boldsymbol{n} \cdot \tilde{\boldsymbol{\xi}}) \hat{\boldsymbol{B}} \\
-\gamma p \nabla \cdot \tilde{\boldsymbol{\xi}}+\boldsymbol{B} \cdot[\tilde{\boldsymbol{Q}}+(\tilde{\boldsymbol{\xi}} \cdot \nabla) \boldsymbol{B}]=\hat{\boldsymbol{B}} \cdot[\nabla \times \tilde{\boldsymbol{A}}+(\tilde{\boldsymbol{\xi}} \cdot \nabla) \hat{\boldsymbol{B}}] .
\end{gathered}
$$

Here $\rho, p, J$, and $B$ denote the equilibrium density, pressure, current, and magnetic field in the plasma region, $\hat{B}$ is the field in the vacuum region, $\tilde{\boldsymbol{Q}}$ is the perturbed field in the plasma, and the vector potential $\tilde{\boldsymbol{A}}$ for the perturbed field outside the plasma satisfies

$$
\nabla \times(\nabla \times \tilde{A})=0 .
$$

Equation (4) provides for continuity of the normal component of the perturbed field and Eq. (5) assures pressure balance across the plasma-vacuum interface. 
Laval et al. [1965] used self adjointness and the constancy of the total energy $\mathcal{E}=K(\partial \overrightarrow{\boldsymbol{\xi}} / \partial t, \partial \tilde{\boldsymbol{\xi}} / \partial t)+\delta W(\tilde{\boldsymbol{\xi}}, \tilde{\boldsymbol{\xi}})$, where

$$
\begin{gathered}
K=\frac{1}{2} \int_{\mathbf{P}} d \tau \rho\left(\frac{\partial \tilde{\boldsymbol{\xi}}}{\partial t}\right)^{2}, \\
\delta W=-\frac{1}{2} \int_{\mathbf{P}} d \tau \tilde{\boldsymbol{\xi}} \cdot \boldsymbol{F}(\tilde{\boldsymbol{\xi}}),
\end{gathered}
$$

to show that the system is stable if, and only if, $\delta W \geq 0$ for all $\tilde{\boldsymbol{\xi}}(\boldsymbol{r}, t)$ that satisfy the boundary conditions, Eqs. (4) and (5). Note that the integral is taken over the plasma region. Bernstein et al. [1958] showed with a few judicious integrations by parts that, for any $\tilde{\xi}$ that satisfies Eqs. (4) and (5),

$$
\delta W_{\mathrm{B}}(\tilde{\boldsymbol{\xi}}, \tilde{\boldsymbol{\xi}})=-\frac{1}{2} \int_{\mathrm{P}} d \tau \tilde{\boldsymbol{\xi}} \cdot \boldsymbol{F}(\tilde{\boldsymbol{\xi}})=\delta W_{\mathrm{P}}+\delta W_{\mathrm{S}}+\delta W_{\mathrm{V}}
$$

where

$$
\begin{array}{lc}
\delta W_{\mathrm{P}}= & \frac{1}{2} \int_{\mathrm{P}} d \tau\left[\tilde{Q}^{2}+\boldsymbol{J} \times \tilde{\boldsymbol{\xi}} \cdot \tilde{\boldsymbol{Q}}+\tilde{\boldsymbol{\xi}} \cdot \nabla p \nabla \cdot \tilde{\boldsymbol{\xi}}+\gamma p(\boldsymbol{\nabla} \cdot \tilde{\boldsymbol{\xi}})^{2}\right], \\
\delta W_{\mathrm{S}}= & \frac{1}{2} \int_{\mathrm{S}} d \sigma(\tilde{\boldsymbol{\xi}} \cdot \boldsymbol{n})^{2}\left[\boldsymbol{n} \cdot \boldsymbol{\nabla}\left(\frac{\hat{\boldsymbol{B}}^{2}}{2}-p-\frac{B^{2}}{2}\right)\right], \\
\delta W_{\mathrm{V}}= & \frac{1}{2} \int_{\mathrm{V}} d \tau|\nabla \times \tilde{\boldsymbol{A}}|^{2} .
\end{array}
$$

We could equally well have omitted the label on $\delta W_{\mathrm{B}}$ here since this evaluation of Eq. (9) is exact for proper $\bar{\xi}$ 's that satisfy Eq. (5). We introduced the label since it can only be considered to be a functional for improper ones. The proof that $\delta W_{\mathrm{B}}(\tilde{\boldsymbol{\xi}}, \tilde{\boldsymbol{\xi}})$ is self adjoint, on which the energy principle is based, relied on the satisfaction of both continuity conditions, Eqs. (4) and (5). Since such a proof for improper $\boldsymbol{\xi}$ 's was not necessary for the demonstration of instability, Bernstein et al. [1958] did not attempt to find one.

A major, but not generally appreciated, contribution of the Energy Principle paper was to show that if one can find an improper $\boldsymbol{\xi}$ that does not satisfy the pressure balance condition of Eq. (5) but makes $\delta W_{\mathrm{B}}(\boldsymbol{\xi}, \boldsymbol{\xi})<0$, then a proper $\tilde{\boldsymbol{\xi}}$ which does satisfy this condition exists such that its $\delta W_{\mathrm{B}}(\tilde{\boldsymbol{\xi}}, \tilde{\boldsymbol{\xi}})$ is also negative. Namely, the authors constructed a slightly modified proper 
displacement $\tilde{\xi}$ that satisfies energy balance for which $\delta W_{\mathrm{B}}$ is changed by an arbitrarily small amount from its value for the improper $\boldsymbol{\xi}$. They set

$$
\tilde{\boldsymbol{\xi}}=\boldsymbol{\xi}+\epsilon \eta \nabla \psi
$$

where $\eta$ is nearly independent of $\psi$ everywhere except in a narrow region between $\psi_{\mathrm{S}}(1-\epsilon)$ and $\psi_{\mathrm{S}}$ at the plasma edge with $\epsilon$ arbitrarily small. In this region it changes rapidly, such that

$$
\epsilon B^{2}|\nabla \psi|^{2} \frac{\partial \eta}{\partial \psi}=O\left(\epsilon^{0}\right)
$$

in the $\boldsymbol{B} \cdot \tilde{\boldsymbol{Q}}$ term in $\delta W_{\mathrm{P}}$. This small modification of the perturbation makes $\tilde{\xi}$ satisfy pressure balance at the plasma-vacuum interface. The change introduces a difference in $\delta W_{\mathrm{B}}$ which consists of only an infinitesimal change in the integrand of $\delta W_{\mathrm{P}}$ in the region $0 \leq \psi \leq \psi_{\mathrm{S}}(1-\epsilon)$ and a finite change in the energy density associated with the torsional Alfvén wave over the shell of negligible thickness $\psi_{\mathrm{S}}(1-\epsilon) \leq \psi \leq \psi_{\mathrm{S}}$. Such a perturbation would create a large current just inside the plasma surface, of order $B(\boldsymbol{n} \cdot \nabla)^{2} \eta \sim \epsilon^{-1}$, which would interact with the local field to produce a stabilizing force. However, this force has been included in the expression for $\delta W_{\mathrm{B}}(\tilde{\boldsymbol{\xi}}, \tilde{\boldsymbol{\xi}})$. It was transformed to the torsional Alfvén wave contribution $\tilde{Q}_{\|}^{2}$ in $\delta W_{\mathrm{P}}$ by an integration by parts in the derivation of Eq. (9), which eliminated one radial derivative on $\tilde{\boldsymbol{\xi}}$ in the perturbed current term $-\tilde{\boldsymbol{\xi}} \cdot(\nabla \times \tilde{\boldsymbol{Q}}) \times \boldsymbol{B}$.

This completes the proof given by Bernstein et al., [1958] that application of the extended energy principle $\delta W_{\mathbf{B}}$ with improper $\boldsymbol{\xi}$ 's provides a rigorous demonstration of the linear stability of the configuration.

Lehnert has incorrectly criticized the extension of the energy principle to unrestricted test functions $\boldsymbol{\xi}$. He chose to work with the functional

$$
\delta W_{\mathrm{L}}=-\frac{1}{2} \int_{\mathrm{P}} d \tau \boldsymbol{\xi} \cdot \boldsymbol{F}(\boldsymbol{\xi})
$$

which differs from $\delta W_{\mathrm{B}}$ by having an additional term from the integration by parts

$$
\begin{gathered}
\delta W_{\mathrm{U}}=\frac{1}{2} \int_{\mathrm{S}} d \sigma(\boldsymbol{\xi} \cdot \boldsymbol{n})\{\{-\gamma p \boldsymbol{\nabla} \cdot \boldsymbol{\xi}+\boldsymbol{B} \cdot \boldsymbol{Q}+\boldsymbol{B} \cdot(\boldsymbol{\xi} \cdot \boldsymbol{\nabla}) \boldsymbol{B} \\
-[\hat{\boldsymbol{B}} \cdot \boldsymbol{\nabla} \times \boldsymbol{A}+\hat{\boldsymbol{B}} \cdot(\boldsymbol{\xi} \cdot \nabla) \hat{\boldsymbol{B}}]\}
\end{gathered}
$$


He then argues that the replacement of the improper $\boldsymbol{\xi}$ by a proper $\tilde{\boldsymbol{\xi}}$ as is done in Eq. (13) actually produces a finite change in $\delta W_{L}$. This is true, but it is important to note that $\delta W_{\mathrm{L}} \neq \delta W_{\mathrm{B}}$ when $\boldsymbol{\xi}$ is improper. The two forms $\delta W_{\mathrm{L}}$ and $\delta W_{\mathrm{B}}$ agree for all proper $\tilde{\boldsymbol{\xi}}$, but not necessarily for improper $\boldsymbol{\xi}$. To get to his version of the extended energy principle, Lehnert then argues that a surface layer current $K$, which must be imposed to provide pressure balance, will add an additional contribution to the force $\boldsymbol{F}(\boldsymbol{\xi})$ in the functional $\delta W_{\mathrm{L}}$, which should be stabilizing. Actually, this additional term,

$$
\delta W_{\mathrm{K}}=-\frac{1}{2} \int_{\mathrm{S}} d \sigma \boldsymbol{\xi} \cdot \boldsymbol{K} \times \boldsymbol{B}
$$

would just cancel the $\delta W_{\mathrm{U}}$ that he apparently lost.

Lehnert's $[1992,1993]$ illustration involves an improper $\boldsymbol{\xi}$ which is taken to be a constant. This perturbation makes $\boldsymbol{F}(\boldsymbol{\xi})=0$ in the body of the plasma, so that, if we ignore $\delta W_{\mathrm{K}}, \delta W_{\mathrm{L}}=0$. An unrestricted rigid displacement $\boldsymbol{\xi}$ thus does not change $\delta W_{\mathrm{L}}$. It contributes to $\delta W_{\mathrm{B}}$, however, through a volume term $\delta W_{\mathrm{P}}$. Modification of this displacement to satisfy pressure balance through Eq. (13) would change $\delta W_{\mathrm{L}}$ by a term of order $\epsilon^{0}$ whereas it makes only an infinitessimal change in $\delta W_{\mathrm{B}}$. Lehnert's addition of $\delta W_{\mathrm{K}}$ properly converts his $\delta W_{\mathrm{L}}$ to $\delta W_{\mathrm{B}}$.

It may be a quite different task to make $\delta W_{B}$ negative than it is to make $\delta W_{\mathrm{L}}$ negative. However, once we have found an improper $\boldsymbol{\xi}$ that makes $\delta W_{\mathrm{B}}$ negative we are done. As noted above, care would have to be taken in the interpretation of $\delta W_{\mathrm{L}}$.

Thus, we can say that Bernstein et al. [1958] have found a "magic" form for $\delta W$, that of Eq. (9), that agrees with $\delta W_{\mathrm{L}}$ over the restricted class of proper test functions $\tilde{\boldsymbol{\xi}}$, but that also has the desirable property that it is sufficiently insensitive to the change $\boldsymbol{\xi} \Rightarrow \tilde{\xi}$ that one can employ it over the unrestricted class of improper $\boldsymbol{\xi}$. The functional $\delta W_{\mathrm{B}}$ is legitimate for determining instability over the extended class of improper $\boldsymbol{\xi}$ 's. It is correct to say that the form $\delta W_{\mathrm{L}}$ does not work without exercising extreme care.

This concludes our defense of the unrestricted energy principle.

In order to endow improper $\boldsymbol{\xi}$ 's with some physical meaning, we must consider what it could mean for an initial perturbation to have a pressure jump. Such a jump would be immediately removed by the plasma. The pressure discontinuity would be spread over a thin layer near the plasma 
vacuum surface much as we have done with the replacement of Eq. (13), $\boldsymbol{\xi} \Rightarrow \tilde{\boldsymbol{\xi}}=\boldsymbol{\xi}+\epsilon \eta \nabla \psi$, where $\eta$ represents the smearing out. However, it is physically sensible to imagine that the plasma is surrounded by a finite surface mass on the plasma-vacuum interface. The pressure jump then moves this surface mass $\hat{\rho}$ as the plasma moves into the vacuum. In this case the energy is still conserved. The kinetic energy is now

$$
K=\frac{1}{2} \int_{\mathrm{P}} d \tau \rho \boldsymbol{v}^{2}+\frac{1}{2} \int_{\mathrm{S}} d \sigma \hat{\rho}(\boldsymbol{v} \cdot \boldsymbol{n})^{2},
$$

while the potential energy is still in the form of the magnetic field and internal pressure energies. This energyr can be expanded in $\boldsymbol{\xi}$ as before. We thus have to second order

$$
\mathcal{E}=\frac{1}{2} \int_{\mathrm{P}} d \tau \rho\left(\frac{\partial \boldsymbol{\xi}}{\partial t}\right)^{2}+\frac{1}{2} \int_{\mathrm{S}} d \sigma \hat{\rho}\left(\frac{\partial \boldsymbol{\xi}}{\partial t} \cdot \boldsymbol{n}\right)^{2}+\delta W(\boldsymbol{\xi}, \boldsymbol{\xi}) .
$$

The equation of motion of the plasma now has two parts:

$$
\rho \frac{\partial^{2} \boldsymbol{\xi}}{\partial t^{2}}=\boldsymbol{F}(\boldsymbol{\xi})
$$

and

$$
\hat{\rho} \frac{\partial^{2} \boldsymbol{\xi}}{\partial t^{2}} \cdot \boldsymbol{n}=F_{0}(\boldsymbol{\xi})
$$

where $F_{0}(\boldsymbol{\xi})$ is the jump in the pressure.

Invoking conservation of energy, we find that the self adjointness property is replaced by

$$
\int_{\mathrm{P}} d \tau \boldsymbol{\xi} \cdot \boldsymbol{F}(\boldsymbol{\eta})+\int_{\mathrm{S}} d \sigma \boldsymbol{\xi} \cdot \boldsymbol{n} F_{0}(\boldsymbol{\eta})=\int_{\mathrm{P}} d \tau \boldsymbol{\eta} \cdot \boldsymbol{F}(\boldsymbol{\xi})+\int_{\mathrm{S}} d \sigma \boldsymbol{\eta} \cdot \boldsymbol{n} F_{0}(\boldsymbol{\xi})
$$

and the potential energy is obtained by setting $\boldsymbol{\eta}=\boldsymbol{\xi}$,

$$
\delta W=-\frac{1}{2} \int_{\mathrm{P}} d \tau \boldsymbol{\xi} \cdot \boldsymbol{F}(\boldsymbol{\xi})-\frac{1}{2} \int_{\mathrm{S}} d \sigma(\boldsymbol{\xi} \cdot \boldsymbol{n})^{2} F_{0}(\boldsymbol{\xi})
$$

Now integration by parts of the $\boldsymbol{\xi} \cdot \boldsymbol{F}(\boldsymbol{\xi})$ integral leads to some extra terms which just cancel the second term in Eq. (23), just as occurred in our analysis of $\delta W_{\mathrm{L}}$. One thus finds that the potential energy in the finite surface mass case is

$$
\delta W=\delta W_{\mathrm{B}}
$$


exactly the expression of Eq. (9) that is usually employed in the energy principle. Thus, $\delta W_{\mathrm{B}}$, defined over the more general improper $\boldsymbol{\xi}$ 's, is still the potential energy! The extra term in this equation is just the work done on the surface to set up the perturbation. It has been pointed out by Lehnert that this term should be included in the energy. But he apparently did not realize that this term was just what is necessary to take into account the extra terms from the integration by parts employed by Bernstein et al. to reach the form $\delta W_{\mathrm{B}}$, if one does not employ pressure balance, Eq. (5).

The question of the meaning of an improper perturbation which minimizes $\delta W$ is important as many stability studies, such as the PEST code [Grimm et al., 1976], determine this minimizing $\boldsymbol{\xi}$ and use it as an indication of the behavior that the system would have with the proper $\tilde{\boldsymbol{\xi}}$. Both of our discussions, of Lehnert's treatment of $\delta W_{\mathrm{L}}$ in which we saw that the $\delta W_{\mathrm{U}}$ term of Eq. (16) just cancels the $\delta W_{K}$ term of Eq. (17) and of the situation where a surface mass was introduced to balance the pressure discontinuity, led to the formulation where the kinetic energy is balanced against $\delta W_{\mathrm{B}}$. Since $\delta W_{\mathrm{B}}$ is used in the PEST formalism, it should be expected from these discussions that the calculations that are made with this code would lead to results that are not far from those using a proper perturbation and the eigenvalues and eigenfunctions should provide an indication of what would be given by a proper normal mode analysis.

\section{Discussion and Summary}

Lehnert $[1991,1992,1993]$ is indeed correct in saying that an improper perturbation $\boldsymbol{\xi}$ that makes the $\delta W_{\mathrm{B}}$ of the extended energy principle, Eq. (9), negative does not in itself correspond to an instability. However, if it exists, a modification from this unstable improper perturbation to a neighboring proper one $\bar{\xi}$ that is unstable and satisfies the pressure balance constraint can be found. This modification of the perturbation produces a large current density very near the plasma-vacuum interface. However, it is wrong to assert that the force associated with this current is not correctly included in the energy balance, $\delta W_{\mathrm{B}}(\tilde{\boldsymbol{\xi}}, \tilde{\boldsymbol{\xi}})=\delta W_{\mathrm{P}}+\delta W_{\mathrm{S}}+\delta W_{\mathrm{V}}$. The integration by parts that led to Eq. (9) has transformed the contribution of this large surface current to the torsional Alfvén wave term $\tilde{Q}_{\|}^{2}$ so that its effects are included in the calculation. 
an improper perturbation leads to the same conclusion. As Lennert notes, 1 this perturbation does not satisfy Eq. (5) by itself, a surface current would have to be introduced to make it do so. This term would make a finite contribution to $\delta W_{\mathrm{L}}$, Eq. (15). However, its contribution would be exactly canceled by surface terms, Eq. (16), which were introduced by the integrations by parts in getting to Eq. (9).

An analysis of a system where a finite surface mass is included to respond to a pressure imbalance at the interface also leads to an extender energy formulation which utilizes the functional $\delta W_{\mathrm{B}}$.

The PEST code [Grimm et al., 1976] was developed to determine the unstable eigenvalues and eigenfunctions in a tokamak by treating improper perturbations associated with the kinetic and potential energies of Eqs. (7) and (9) but not necessarily satisfying the boundary condition of Eq. (5). Since neither the destabilizing surface term arising from the integration over the plasma volume nor the stabilization associated with a surface current or an acceleration at the plasma-vacuum interface was incorporated into the code, it should produce a close approximation to what would have been calculated with a proper perturbation. The results from PEST are in good agreement with those given by a nonvariational code NOVA [Cheng and Chance, 1987], which evaluates the eigenfunctions directly from the force balance equations while incorporating both of the constraints of Eqs. (4)and (5) on the normal component of the perturbed field and pressure balance at the plasma-vacuum interface.

This note demonstrates the difficulties that can be encountered when one attempts to utilize mixed methods of looking simultaneously at both forces and energies to treat a problem. A direct calculation of the forces, as is done by the NOVA code, and a variational study using the energy principle, characterized by the PEST code, give the same results. An attempt to calculate both forces and energies must be done with care to ensure that the same terms are not counted twice. In any case, a proper unstable perturbation which is close to an improper one that makes $\delta W_{\mathrm{B}}$ of Eq. (9) negative can be constructed by means of Eq. (13). This shows that application of the "extended energy principle" of Bernstein et al. [1958] provides a definitive determination of the stability properties of a system.

Acknowledgements - We are indebted to Dr. P. H. Rutherford for calling 
this problem to our attention and noting the importance of preparing this note. We also thank Professor B. Lehnert for his kind and helpful reply when we sent him a preliminary version. The work is funded by the US Department of Energy under contract No. DE-AC02-76-CHO-3073 with Princeton University. 


\section{References}

${ }^{1}$ Bernstein, I. B., Frieman,.E. A., Kruskal, M. D., and Kulsrud, R. M. (1958) Proc. Roy. Soc. (London) A244, 17

${ }^{2}$ Cheng, C. Z., and Chance, M. S. (1987) J. Comp. Phys. 71, 124.

${ }^{3}$ Grimm, R. C., Greene, J. M., and Johnson, J. L. (1976) Methods in Computational Physics (Academic Press, N. Y.) Vol. 16, p. 253.

${ }^{4}$ Lehnert, B. (1991) Plasma Phys. Control Fusion 33, 1757

${ }^{5}$ Lehnert, B. (1992) Plasma Phys. Control Fusion 34, 1651

${ }^{6}$ Lehnert, B. (1993) Plasma Phys. Control Fusion 35, 873

${ }^{7}$ Laval, G., Mercier, C, and Pellat, R. M. (1965) Nuclear Fusion 5, 156 


\section{EXTERNAL DISTRIBUTION IN ADDITION TO UC-420}

Dr. F. Paoloni, Univ. of Wollongong, AUSTRALIA

Prof. M.H. Brennan, Univ. of Sydney, AUSTRALIA

Plasma Research Lab., Australian Nat. Univ., AUSTRALIA

Prof. I.R. Jones, Flinders Univ, AUSTRALIA

Prof. F. Cap, Inst. for Theoretical Physics, AUSTRIA

Prof. M. Heindler, Institut für Theoretische Physik, AUSTRIA

Prof. M. Goossens, Astronomisch Instituut, BELGIUM

Ecole Royale Militaire, Lab. de Phy. Plasmas, BELGIUM

Commission-European, DG. XII-Fusion Prog., BELGIUM

Prof. R. Bouciqué, Rijksuniversiteit Gent, BELGIUM

Dr. P.H. Sakanaka, Instituto Fisica, BRAZIL

Prof. Dr. I.C. Nascimento, Instituto Fisica, Sao Paulo, BRAZIL Instituto Nacional De Pesquisas Espaciais-INPE, BRAZIL.

Documents Office, Atomic Energy of Canada Lid., CANADA

Ms. M. Morin, CCFMTokamak de Varennes, CANADA

Dr. M.P. Bachynski, MPB Technologies, Inc., CANADA

Dr. H.M. Skarsgard, Univ. of Saskatchewan, CANADA

Prof. J. Teichmann, Univ. of Montreal, CANADA

Prof. S.R. Sreenivasan, Univ. of Calgary, CANADA

Prof. T.W. Johnston, INRS-Energie, CANADA

Dr. R. Bolton, Centre canadien de fusion magnétique, CANADA

Dr. C.R. James, Univ. of Alberta, CANADA

Dr. P. Lukác, Komenského Universzita, CZECHO-SLOVAKIA

The Librarian, Culham Laboratory, ENGLAND

Library, R61, Rutherford Appleton Laboratory, ENGLAND

Mrs. S.A. Hutchinson, JET Library, ENGLAND

Dr. S.C. Sharma, Univ. of South Pacific, FIJI ISLANDS

P. Mähönen, Univ. of Helsinki, FINLAND

Prof. M.N. Bussac, Ecole Polytechnique, FRANCE

C. Mouttet, Lab. de Physique des Milieux lonisés, FRANCE

J. Radet, CEN/CADARACHE - Bat 506, FRANCE

Prof. E. Economou, Univ. of Crete, GREECE

Ms. C. Rinni, Univ. of loannina, GREECE

Preprint Library, Hungarian Academy of Sci., HUNGARY

Dr. B. DasGupta, Saha Inst. of Nuclear Physics, INDIA

Dr. P. Kaw, Inst. for Plasma Research, INDIA

Dr. P. Rosenau, Israel Inst. of Technology, ISRAEL

Librarian, International Center for Theo Physics, ITALY

Miss C. De Palo, Associazione EURATOM-ENEA, ITALY

Dr. G. Grosso, Istituto di Fisica del Plasma, ITALY

Prof. G. Rostangni, Istituto Gas lonizzati Del Cnr. ITALY
Dr. H. Yamato, Toshiba Res \& Devel Center, JAPAN

Prof. I. Kawakami, Hiroshima Univ., JAPAN

Prof. K. Nishikawa, Hiroshima Univ., JAPAN

Librarian, Naka Fusion Research Establishment, JAERI, JAPAN

Director, Japan Atomic Energy Research Inst., JAPAN

Prof. S. Itoh, Kyushu Univ., JAPAN

Research Info. Ctr., National Instit. for Fusion Science, JAPAN

Prof. S. Tanaka, Kyoto Univ., JAPAN

Library, Kyoto Univ., JAPAN

Prof. N. Inove, Univ. of Tokyo, JAPAN

Secretary, Plasma Section, Electrotechnical Lab., JAPAN

S. Mori, Technical Advisor, JAERI, JAPAN

Dr. O. Mitarai, Kumamoto Inst. of Technology, JAPAN

Dr. G.S. Lee, Korea Basic Sci. Ctr., KOREA

J. Hyeon-Sook, Korea Atomic Energy Research Inst., KOREA

D.I. Choi, The Korea Adv. Inst. of Sai. \& Tech., KOREA

Prof. B.S. Liley, Univ. of Waikato, NEW ZEALAND

Inst of Physics, Chinese Acad Sci PEOPLE'S REP. OF CHINA Library, Inst. of Plasma Physics, PEOPLE'S REP. OF CHINA

Tsinghua Univ. Library, PEOPLE'S REPUBLIC OF CHINA

2. Li, S.W. Inst Physics, PEOPLE'S REPUBLIC OF CHINA

Prof. J.A.C. Cabral, Instituto Superior Tecnico, PORTUGAL

Prof. M.A. Hellberg, Univ. of Natal, S. AFRICA

Prof. D.E. Kim, Pohang Inst. of Sci. \& Tech., SO. KOREA

Prot. C.I.E.M.A.T, Fusion Division Librany, SPAIN

Dr. L. Stenflo, Univ. of UMEA, SWEDEN

Library, Royal Inst. of Technology, SWEDEN

Prof. H. Wilhelmson, Chalmers Univ. of Tech., SWEDEN

Centre Phys. Des Plasmas, Ecole Polytech, SWITZERLAND

Bibliotheek, Inst. Voor Plasma-Fysica, THE NETHERLANDS

Asst. Prof. Dr. S. Cakir, Middle East Tech. Univ., TURKEY

Dr. V.A. Giukhikh,Sci. Res. Inst. Electrophys.I Apparatus, USSR

Dr. D.D. Ryutov, Siberian Branch of Academy of Sci., USSR

Dr. G.A. Eliseev, I.V. Kurchatov Inst., USSR

Librarian, The Ukr.SSR Academy of Sciences, USSR

Dr. L.M. Kovrizhnykh, inst. of Gencral Physics, USSR Kernforschungsanlage GmbH, Zentralbibliothek, W. GERMANY

Bibliothek, Inst. Für Plasmaforschung, W. GERMANY

Prof. K. Schindler, Ruhr-Universitát Bochum, W. GERMANY

Dr. F. Wagner, (ASDEX), Max-Planck-Institut, W. GERMANY

Librarian, Max-Planck-Institut, W. GERMANY 

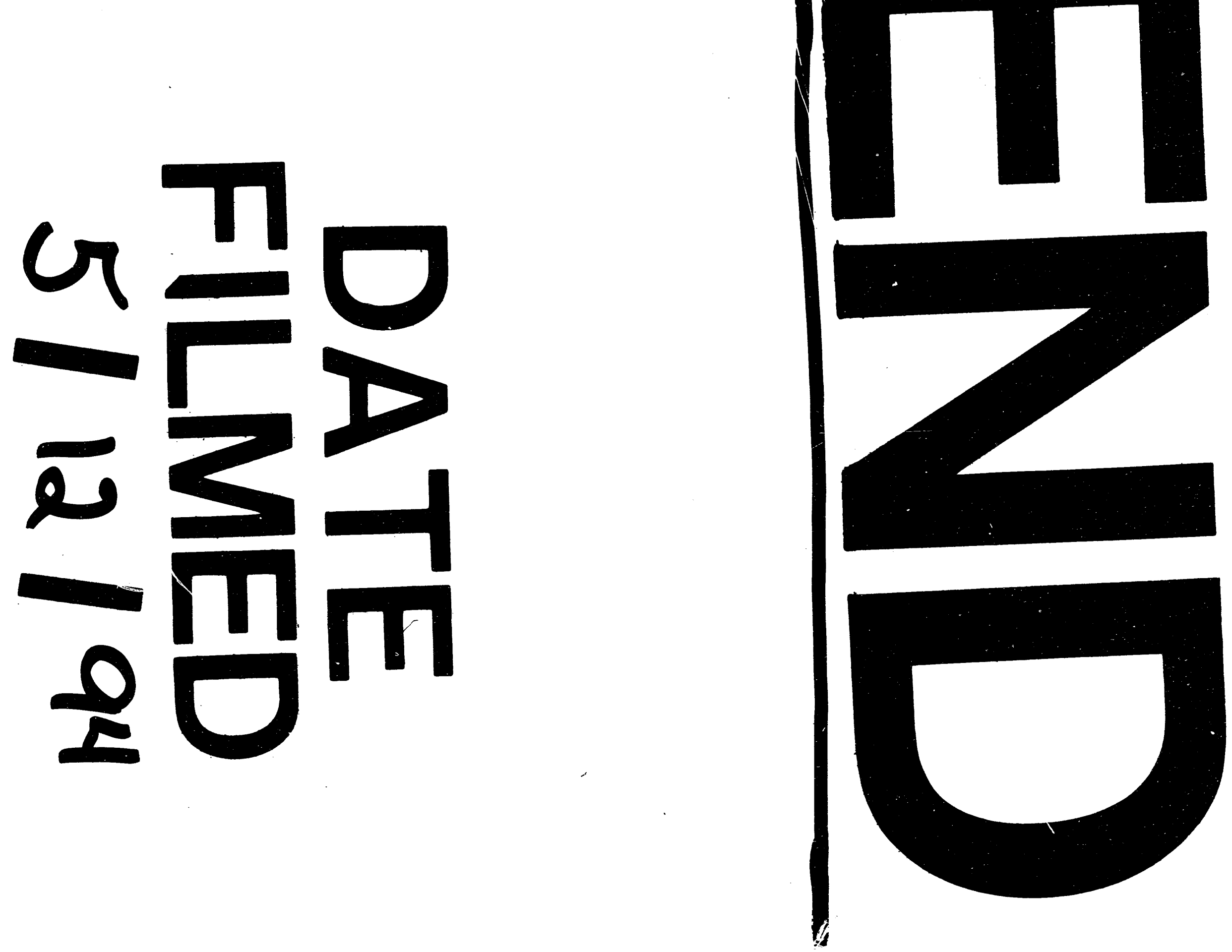
